\title{
Micro-computed tomography: A novel diagnostic technique for the evaluation of gastrointestinal specimens $\square$
}

\section{(ㄷ)(1) $\odot$}

Authors

Noboru Kawata ${ }^{1,2}$, Alexei Teplov ${ }^{1}$, Peter Ntiamoah ${ }^{1}$, Jinru Shia ${ }^{1}$, Meera Hameed ${ }^{1}$, Yukako Yagi ${ }^{1}$

Institutions

1 Department of Pathology, Memorial Sloan Kettering Cancer Center, New York, New York, United States

2 Division of Endoscopy, Shizuoka Cancer Center, Shizuoka, Japan

submitted 25.1.2021

accepted after revision 23.6.2021

\section{Bibliography}

Endosc Int Open 2021; 09: E1886-E1889

DOI 10.1055/a-1546-8063

ISSN 2364-3722

(c) 2021. The Author(s).

This is an open access article published by Thieme under the terms of the Creative Commons Attribution-NonDerivative-NonCommercial License, permitting copying and reproduction so long as the original work is given appropriate credit. Contents may not be used for commercial purposes, or adapted, remixed, transformed or built upon. (https://creativecommons.org/licenses/by-nc-nd/4.0/)

Georg Thieme Verlag KG, Rüdigerstraße 14,

70469 Stuttgart, Germany

\section{Corresponding author}

Noboru Kawata, MD, Department of Pathology, Memorial Sloan Kettering Cancer Center, 1275 York Avenue, New York, NY, 10065 United States

n.kawata@scchr.jp

\section{ABSTRACT}

Micro-computed tomography (micro-CT) is a non-destructive modality that can be used to obtain high-resolution three-dimensional (3D) images of the whole sample tissue; the usefulness of micro-CT has been reported for evaluation of breast cancer and lung cancer. However, this novel diagnostic technique has never been used for evaluating endoscopically resected gastrointestinal specimens. In the present study, we scanned 13 formalin-fixed paraffin-embedded (FFPE) tissue blocks of a normal human colon and gastric tissue samples using micro-CT. The evaluation comprised a comparison of the acquired whole block images with the images of the corresponding cross-sectional slice of the hematoxylin and eosin-stained slide. Micro-CT was able to produce images of the whole sample and clearly depict tissues such as glandular structures, muscularis mucosae, and blood vessels in the FFPE tissue blocks of normal gastrointestinal samples. Furthermore, the $3 \mathrm{D}$ reconstructed could be used to create a cross-sectional image and reflected the surface structure of samples obtained from any site. Micro-CT has the potential to become a highly promising pathological diagnostic assistance tool for endoscopically resected gastrointestinal specimens in combination with conventional microscopic examination.

\section{Introduction}

Accurate pathological diagnosis of endoscopically resected gastrointestinal cancer is essential for the determination of the postoperative management [1-3]. The current pathological diagnosis of gastrointestinal cancer involves the examination of two-dimensional (2D) cross-sectional images of the resected specimens; thus, only a small part of the actual tumor is assessed. Micro-computed tomography (micro-CT) is a threedimensional (3D) x-ray imaging technique, similar to medical $\mathrm{CT}$ that provides $3 \mathrm{D}$ images on a smaller scale and at a higher resolution. Previous reports demonstrated that whole block imaging (WBI) of specimens in formalin-fixed paraffin-embedded (FFPE) tissue blocks could be obtained at a microscopic level using micro-CT $[4,5]$. This technology has the potential to significantly impact the pathological evaluations of endoscopically resected gastrointestinal cancers. Therefore, we assessed whether individual tissue structures of the normal gastrointestinal tissue samples obtained using micro-CT could be identified on wide-band imaging (WBI) as the preliminary investigation of its application for the pathological diagnosis of endoscopically resected gastrointestinal cancers.

\section{Materials and methods}

A total of 18 samples (four from normal colonic mucosa [instead of the endoscopically resected specimens], five from normal colonic walls, and nine from normal gastric walls) were collected and analyzed from 13 surgically resected human specimens between May 2018 and July 2019. The tissue samples ob- 
tained from the colonic and gastric wall and colonic mucosa were converted into nine FFPE tissue blocks $(1 \times 2$-inch cassettes) and four whole-mount FFPE tissue blocks $(2 \times 3$-inch cassettes), respectively. The FFPE tissue blocks were scanned using a custom-built micro-CT scanner ( Fig. 1) (Nikon Metrology NV, Leuven, Belgium), as per a previously described protocol [4]. The image resolution of WBI depends primarily on the specimen size; therefore, the entire specimen was initially scanned at a low resolution (pixel size: 10 to $26 \mu \mathrm{m}$ ); thereafter, we performed high-magnification and high-resolution scanning of the region of interest (ROI) within the specimen (pixel size: 5 to $9 \mu \mathrm{m})$. The data obtained were reconstructed using the CT Agent Medical Alpha software (Nikon Metrology NV, Leuven, Belgium). The reconstructed imaging data were then visualized and analyzed using commercially available software, including Dragonfly (Object Research Systems, Montreal, Canada) and VGStudio Max (Volume Graphics, Heidelberg, Germany). Finally, each tissue structure from the WBI was assessed to check how closely it matched the corresponding cross-sectional slice image of the whole slide imaging (WSI) of hematoxylin and eosin (H\&E)-stained slide, which was scanned using NanoZoomer 2.0HT and S60 (Hamamatsu Photonics, Shizuoka, Japan). This study protocol was approved by the institutional review board of Memorial Sloan Kettering Cancer Center.

\section{Results}

Thirteen FFPE tissue blocks were scanned 25 times with the following settings: $70 \mathrm{kV}$ peak voltage, $7.0 \mathrm{~W}, 4821$ projections, four-frame average per projection distributed over $360^{\circ}$, and an exposure time of 2500 to $3250 \mathrm{~ms}$ per frame. The average scanning duration was 23 hours per sample. The corresponding cross-sectional slice images of WBI and WSI of H\&E-stained slides of a colonic wall are shown in $>$ Fig. 2. The grayscale WBI images acquired initially were digitally colored to mimic the H\&E-stained slide images. The WBI images were evaluated as serial cross-sectional images ( $\triangleright$ Video $\mathbf{S} 1$ ). A comparison between the WBI and WSI of H\&E-stained slide images revealed that tissue structures, such as the glandular structures, muscularis mucosae, and blood vessels were clearly visualized, with each layer of the wall was distinguishable. Each tissue image in WBI could be correlated one-to-one with the WSI of H\&Estained slide images. The WBI of gastrointestinal tissues with a pixel size of $5 \mu \mathrm{m}$ was almost equivalent to the microscope image at $4 \times$ objective magnification. Furthermore, the $3 \mathrm{D}$ reconstructed images could be sectioned and rotated in different directions, producing cross-sectional images of any site ( $>$ Fig. $\mathbf{3}$, - Video S2), hence providing a better understanding of the vascular pattern and positional relationship between all the tissues.

\section{Discussion}

This pilot study evaluated the WBI of normal human gastrointestinal samples in FFPE tissue blocks. Our study showed that micro-CT could provide serial cross-sectional and 3D images of the entire sample with the images close to those with H\&E-

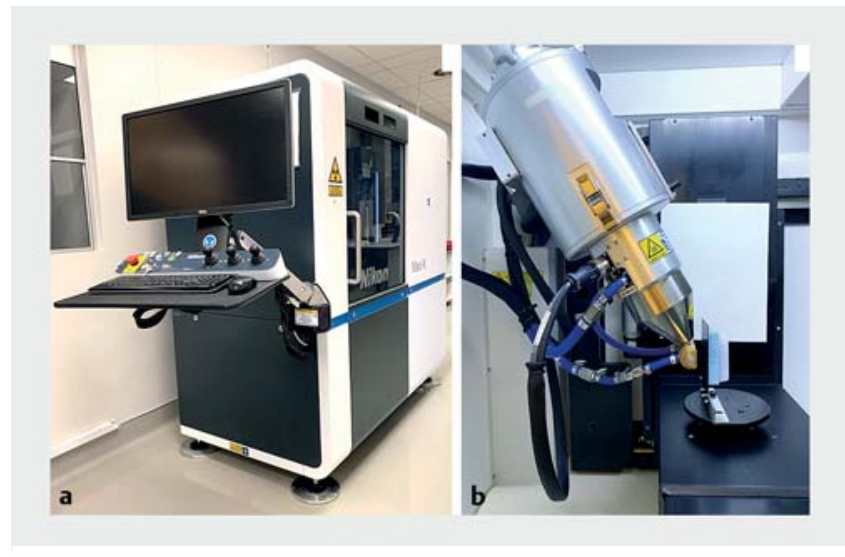

- Fig. 1 a Micro-CT scanner (Nikon Metrology NV, Leuven, Belgium). b Micro-CT scanning for whole-mount FFPE tissue block. CT, computed tomography; FFPE, formalin-fixed paraffin-embedded.

stained slides in a non-destructive manner. Furthermore, the individual tissue structures of normal gastrointestinal tissue clearly visualized and could be almost completely correlated with those in the H\&E-stained slide images.

The advantage of using WBI to evaluate the entire specimen is that it provides additional pathological information along with the conventional diagnosis. In the present study, we could identify each tissue through one-to-one correspondence between the images of WBI and H\&E-stained slides. Therefore, WBI may distinguish the tumor cells from the non-tumor cells and identify the actual deepest point of invasion of the gastrointestinal cancer that is difficult to determine with conventional pathological diagnosis methods. As a result, the conventional pathological diagnosis and curability of endoscopically resected specimens may be changed by WBI. Moreover, the non-destructive identification of the ROI of the resected specimen is useful for making the decision regarding the cutting line for the H\&E-stained slide section. Thus, we believe that the use of micro-CT in combination with the conventional pathological methods may improve the efficiency of the pathological diagnosis of gastrointestinal cancer and processing in the future.

The $3 \mathrm{D}$ images make it easier to understand the vascular pattern, horizontal spread pattern of the tumor, and the spatial relationship between each tissue, which are otherwise difficult to understand using conventional 2D slide images. Moreover, surface structures and cross-sectional images of resected specimens can be obtained for any location; this facilitates the one-to-one correspondence of the images between preoperative endoscopy and the pathology of the resected specimens. Further, by reconstructing $3 \mathrm{D}$ blood vessel images [5], it may be possible to correlate the microvascular pattern of the tumor between pathology and magnified endoscopic images with narrow-band imaging. Therefore, micro-CT is expected to contribute not only to immensely toward aiding pathological diagnosis but also to further improvement of the endoscopic diagnosis.

Recently, several techniques for generating high-resolution $3 \mathrm{D}$ images of gastrointestinal specimens have been reported 

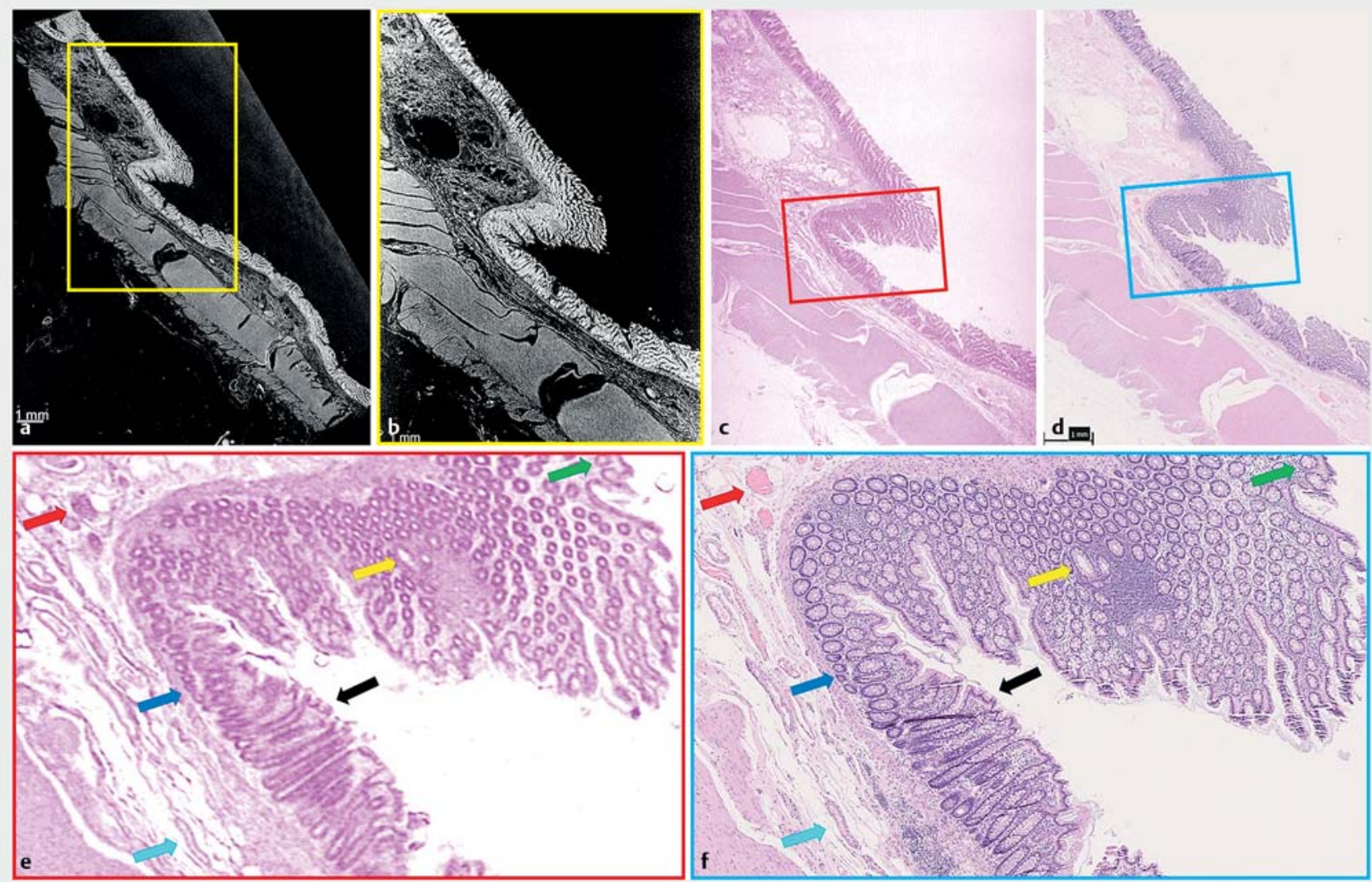

Fig. 2 Comparison of WBI of a FFPE tissue block of a normal colonic wall with the corresponding slice of WSI of the H\&E-stained slide. a WBI of the entire sample (pixel size $11 \mu \mathrm{m}$ ). b Grayscale WBI of the yellow frame in a visualized using Dragonfly (pixel size $5 \mu \mathrm{m}$ ). c Digitally stained WBI of $\mathbf{b}$ visualized using Dragonfly. $\mathbf{d}$ A matching slice of WSI of H\&E-stained slide. e Zoomed image of the red frame in $\mathbf{c}$. $\mathbf{f}$ Zoomed image of the blue frame in d. Arrows indicate the corresponding tissue between WBI and WSI of the H\&E-stained slide image. WBI, whole block imaging; FFPE, formalin-fixed paraffin-embedded; WSI, whole slide imaging; H\&E, hematoxylin and eosin.

\section{DIDEO}

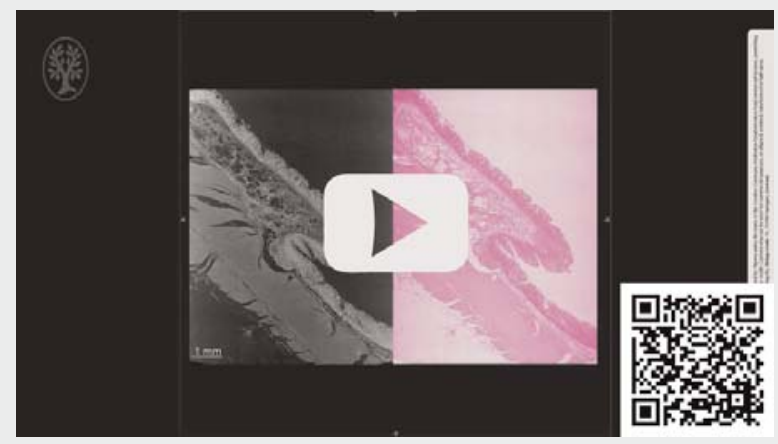

- Video S1 Serial cross-sectional whole block image of the colonic wall created using Dragonfly.

$[6,7]$. However, these methods require special preparations, such as sectioning, staining, and deparaffinization for creating $3 \mathrm{D}$ images. The advantage of micro-CT scanning compared to those of previous methods is that micro-CT does not require these scanning preparations, and conventional FFPE tissue blocks can be scanned as they are. Thus, the tissue can be preserved for subsequent evaluations. Moreover, there is no radiation damage to protein expression [4] and micro-CT scanning does not hinder conventional pathological assessment. Therefore, we believe that the evaluation of endoscopic resected specimen by WBI can be applied in clinical practice in the near future.

This study has some limitations. First only a few FFPE tissue blocks of normal gastrointestinal specimens were evaluated. Second, the resolution of micro-CT was not sufficient for the visualization of nuclei and certain kinds of cells. However, our results strongly support future research on the use of micro-CT for endoscopically resected gastrointestinal cancers.

\section{Conclusions}

In conclusion, micro-CT could provide WBI, enabling clear visualization and evaluation of FFPE tissue blocks of normal human gastrointestinal tissue samples, similar to a microscopic examination. The combination of micro-CT with conventional diagnostic methods shows promise as a pathological diagnostic assistance tool for endoscopically resected gastrointestinal specimens. 

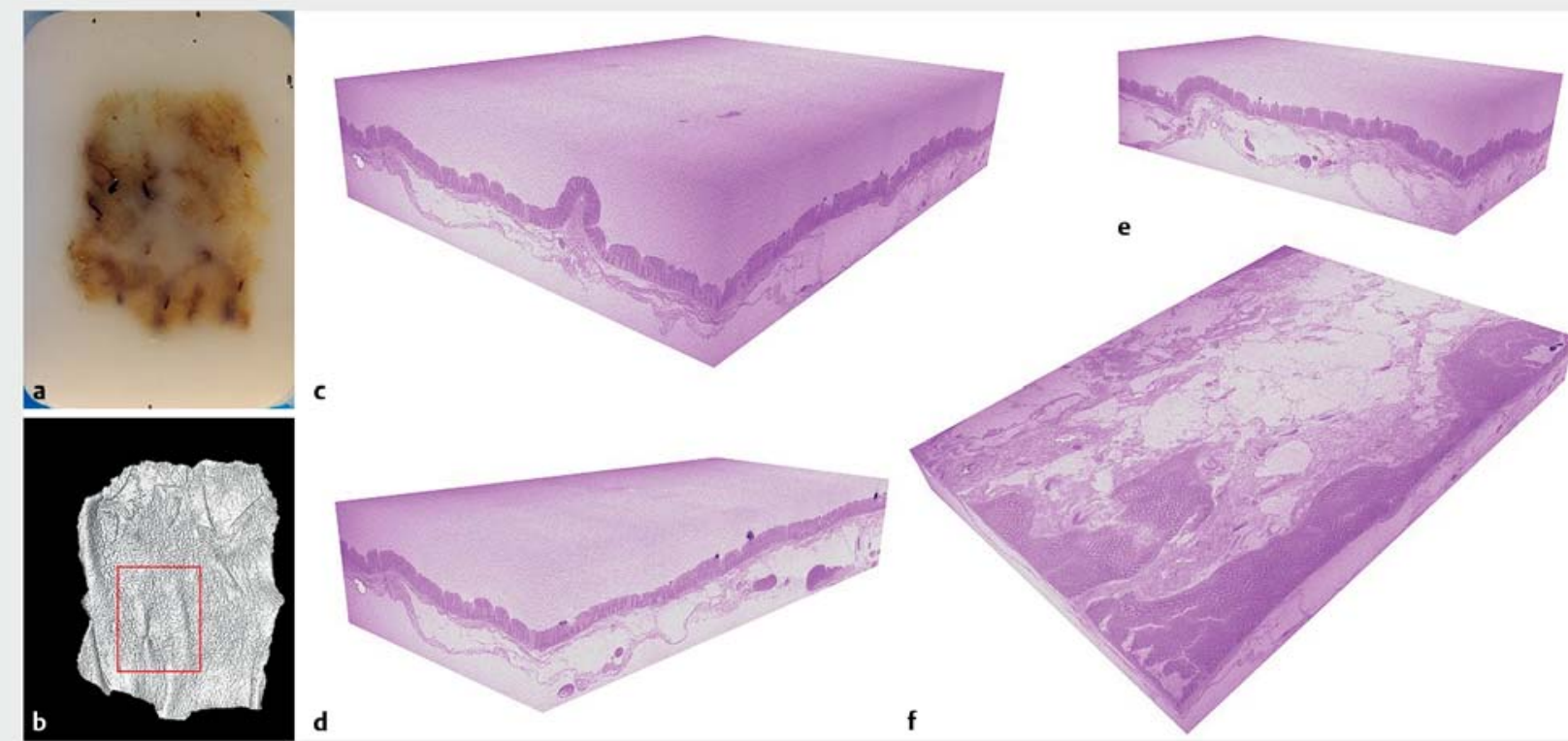

e
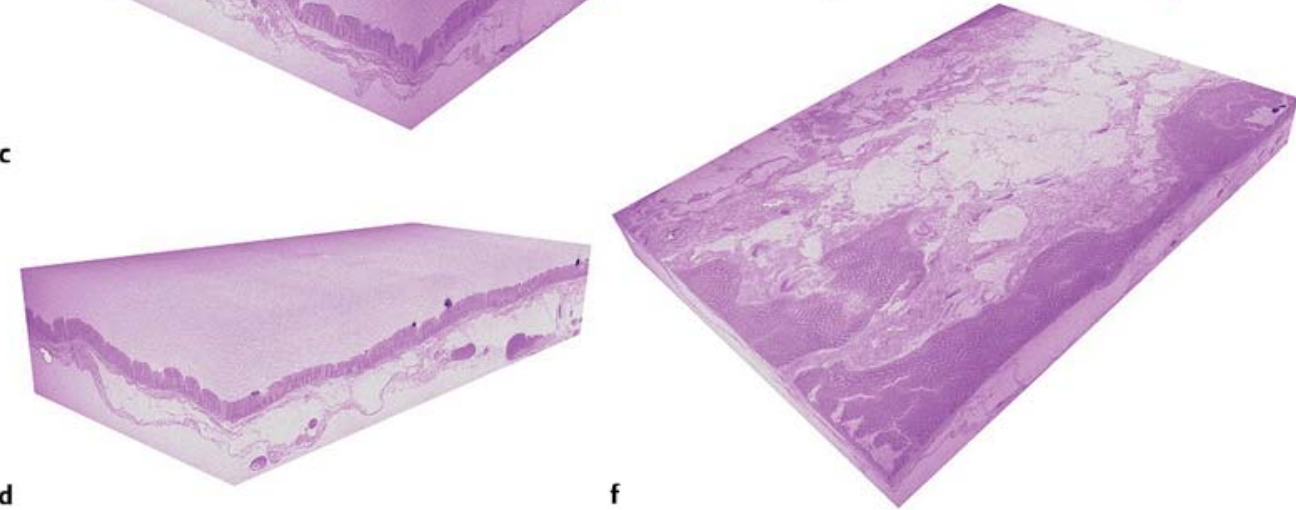

Fig. 3 Three-dimensional WBI of the whole-mount FFPE tissue block of a normal colon mucosa visualized using VG Studio Max. a Wholemount FFPE tissue block of a normal colon mucosa ( $2 \times 3$-inch cassettes). $\mathbf{b} 3 \mathrm{D}$ WBI of the whole-mount FFPE tissue block of a normal colon mucosa (voxel size $23 \mu \mathrm{m}$ ). c $3 \mathrm{D}$ WBI of the red frame of $\mathbf{b}$ with digital staining (voxel size $9 \mu \mathrm{m}$ ). $\mathbf{d}$ Sagittal plane of $\mathbf{c}$. e Coronal plane of $\mathbf{c}$. f Transverse plane of $\mathbf{c}$. FFPE, formalin-fixed paraffin-embedded; $3 \mathrm{D}$, three-dimensional.

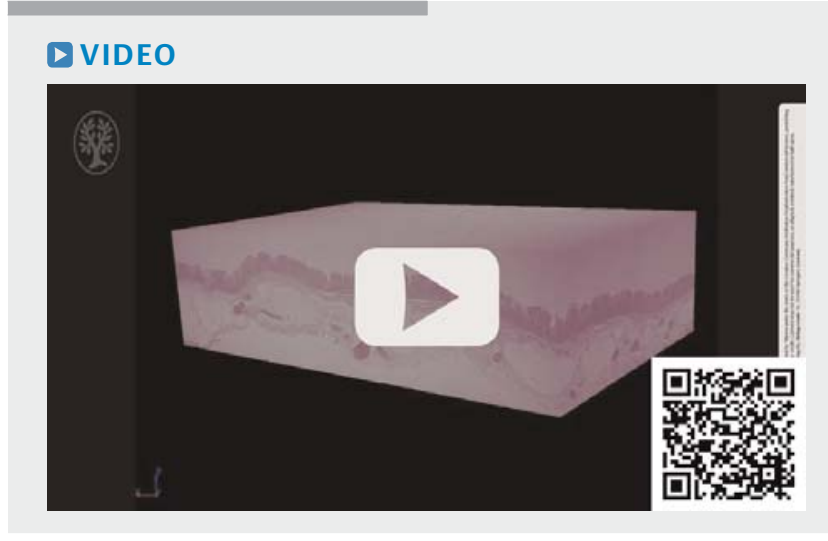

- Video $\mathbf{S 2}$ Three-dimensional whole block image of colon mucosa created using VG Studio Max.

\section{Acknowledgements}

The authors wish to acknowledge Nikon Metrology NV, The Warren Alpert Foundation Center for Digital and Computational Pathology at the Memorial Sloan Kettering Cancer Center, Makoto Nishimura, Kareem S. Ibrahim, Fadi Odeh, and Christina M. Virgo, who supported our research. This study would not have been possible without their support and contributions; it is greatly appreciated.

\section{Competing interests}

The authors declare that there are no conflicts of interest.

\section{References}

[1] Japanese Gastric Cancer Association. Japanese gastric cancer treatment guidelines 2014 (ver. 4). Gastric Cancer 2017; 20: 1-19

[2] Ono H, Yao K, Fujishiro M et al. Guidelines for endoscopic submucosal dissection and endoscopic mucosal resection for early gastric cancer. Dig Endosc 2016; 28: 3-15

[3] Hashiguchi Y, Muro K, Saito Y et al. Japanese Society for Cancer of the Colon and Rectum (JSCCR) guidelines 2019 for the treatment of colorectal cancer. Int J Clin Oncol 2020; 25: 1-42

[4] Teplov A, Tabata K, Fu X et al. Development of standard operating procedure (SOP) of micro-computed tomography (micro-CT) in pathology. Diagn Pathol 2019; 5: 273

[5] Scott AE, Vasilescu DM, Seal KA et al. Three dimensional imaging of paraffin embedded human lung tissue samples by micro-computed tomography. PLoS One 2015; 10: e0126230

[6] Mizutani H, Ono S, Ushiku T et al. Transparency-enhancing technology allows three-dimensional assessment of gastrointestinal mucosa: A porcine model. Pathol Int 2018; 68: 102-108

[7] Ueda T, Morita K, Koyama F et al. A detailed comparison between the endoscopic images using blue laser imaging and three-dimensional reconstructed pathological images of colonic lesions. PLoS One 2020; 15: e0235279 